\title{
Pacific
}

Journal of

Mathematics

\section{INVARIANCE OF THE BFV COMPLEX}

FLORIAN SCHÄTZ 


\title{
INVARIANCE OF THE BFV COMPLEX
}

\author{
FLORIAN SCHÄTZ
}

The Batalin-Vilkovisky-Fradkin (BFV) formalism, introduced to handle classical systems equipped with symmetries, associates a differential graded Poisson algebra to any coisotropic submanifold $S$ of a Poisson manifold $(M, \Pi)$. However, the assignment given by mapping a coisotropic submanifold to a differential graded Poisson algebra is not canonical since in the construction several choices have to be made. One has to fix an embedding of the normal bundle $N S$ of $S$ into $M$ as a tubular neighborhood, a connection $\nabla$ on $N S$, and a special element $\Omega$.

We show that different choices of a connection and an element $\Omega-$ but with the tubular neighborhood fixed - lead to isomorphic differential graded Poisson algebras. If the tubular neighborhood is changed as well, invariance can still be restored at the level of germs.

\section{Introduction}

The Batalin-Vilkovisky-Fradkin (BFV) complex was introduced to understand physical systems with complicated symmetries [Batalin and Fradkin 1983; Batalin and Vilkovisky 1977]. The connection to homological algebra was made explicit in [Stasheff 1997] later on. We focus on the smooth setting; that is, we want to consider arbitrary coisotropic submanifolds of smooth finite-dimensional Poisson manifolds. Bordemann [2000] and Herbig [2006] found a convenient adaptation of the BFV construction in this framework: One obtains a differential graded Poisson algebra associated to any coisotropic submanifold. In [Schätz 2009a], a slight modification of the construction of Bordemann and Herbig was presented. It made use of the language of higher homotopy structures and provided in particular a conceptual construction of the BFV bracket.

In the smooth setting, the construction of the BFV complex requires a choice of the following data: (1) an embedding of the normal bundle of the coisotropic

MSC2000: primary 53D17, 55U99; secondary 17B60.

Keywords: Poisson geometry, coisotropic submanifolds, BFV complex, homological algebra.

Partially supported by the Institute of Mathematics, University Zurich, by a research grant of the University Zurich, by the SNF-grant 200020-121640/1, by the European Union through the FP6 Marie Curie RTN ENIGMA (contract number MRTN-CT-2004-5652), and by the European Science Foundation through the MISGAM program. 
submanifold as a tubular neighborhood into the ambient Poisson manifold, (2) a connection on the normal bundle, and (3) a special function on a smooth graded manifold, called a BFV charge.

We apply the point of view established in [Schätz 2009a] to clarify the dependence of the resulting BFV complex on these data. If one leaves the embedding fixed and only changes the connection and the BFV charge, one simply obtains two isomorphic differential graded Poisson algebras; see Theorem 3.4. Note that the dependence on the choice of BFV charge was already well understood; see [Stasheff 1997], for instance. Dependence on the embedding is more subtle. In Definition 4.2, we introduce the notion of restriction of a given BFV complex to an open neighborhood of the coisotropic submanifold inside its normal bundle, and in Theorem 4.4 we show that different choices of embeddings lead to isomorphic restricted BFV complexes. Corollary 4.6 says that a germ version of the BFV complex is independent of all the choices up to isomorphism.

As it turns out, the differential graded Poisson algebra associated to a fixed embedding of the normal bundle as a tubular neighborhood yields a description of the moduli space of coisotropic sections in terms of the BFV complex; see [Schätz 2009b].

\section{Preliminaries}

The purpose of this section is threefold: to recollect some facts about the theory of higher homotopy structures, to recall some concepts concerning Poisson manifolds and coisotropic submanifolds, and to outline the construction of the BFV complex. More details on these subjects can be found in [Schätz 2009a, Sections 2 and 3] and in the references therein. We assume the reader to be familiar with the theory of graded algebras and smooth graded manifolds.

2a. L L-algebras: Homotopy transfer and homotopies. Let $V$ be a $\mathbb{Z}$-graded vector space over $\mathbb{R}$ (or any other field of characteristic 0 ); that is, $V$ is a collection $\left(V_{i}\right)_{i \in \mathbb{Z}}$ of vector spaces $V_{i}$ over $\mathbb{R}$. The homogeneous elements of $V$ of degree $i \in \mathbb{Z}$ are the elements of $V_{i}$. We denote the degree of a homogeneous element $x \in V$ by $|x|$. A morphism $f: V \rightarrow W$ of graded vector spaces is a collection $\left(f_{i}: V_{i} \rightarrow W_{i}\right)_{i \in \mathbb{Z}}$ of linear maps. The $n$-th suspension functor $[n]$ from the category of graded vector spaces to itself is defined as follows: Given a graded vector space $V$, we denote by $V[n]$ the graded vector space corresponding to the collection $V[n]_{i}:=V_{n+i}$. The $n$-th suspension of a morphism $f: V \rightarrow W$ of graded vector spaces is given by the collection $\left(f[n]_{i}:=f_{n+i}: V_{n+i} \rightarrow W_{n+i}\right)_{i \in \mathbb{Z}}$. The tensor product of two graded vector spaces $V$ and $W$ over $\mathbb{R}$ is the graded vector 
whose component in degree $k$ is given by

$$
(V \otimes W)_{k}:=\bigoplus_{r+s=k} V_{r} \otimes W_{s} .
$$

The denote this graded vector space by $V \otimes W$.

The structure of a flat $L_{\infty}$ [1]-algebra on $V$ is given by a family of multilinear maps $\left(\mu^{k}: V^{\otimes k} \rightarrow V[1]\right)_{k \geq 1}$ such that

(1) $\mu^{k}(\cdots \otimes a \otimes b \otimes \cdots)=(-1)^{|a||b|} \mu^{k}(\cdots \otimes b \otimes a \otimes \cdots)$ holds for all $k \geq 1$ and all homogeneous elements $a$ and $b$ of $V$;

(2) the family of Jacobiators $\left(J^{k}\right)_{k \geq 1}$ defined by

$$
\begin{aligned}
& J^{k}\left(x_{1} \cdots x_{n}\right) \\
& \quad:=\sum_{r+s=k} \sum_{\sigma \in(r, s) \text {-shuffles }} \operatorname{sign}(\sigma) \mu^{s+1}\left(\mu^{r}\left(x_{\sigma(1)} \otimes \cdots \otimes x_{\sigma(r)}\right) \otimes x_{\sigma(r+1)} \otimes \cdots \otimes x_{\sigma(n)}\right),
\end{aligned}
$$

vanishes identically. Here $\operatorname{sign}(\cdot)$ is the Koszul sign, that is, the representation of $\Sigma_{n}$ on $V^{\otimes n}$ induced by mapping the transposition $(2,1)$ to $a \otimes b \mapsto$ $(-1)^{|a||b|} b \otimes a$, and $(r, s)$-shuffles are permutations $\sigma$ of $\{1, \ldots, k=r+s\}$ such that $\sigma(1)<\cdots<\sigma(r)$ and $\sigma(r+1)<\cdots<\sigma(k)$.

Since we are only going to consider flat $L_{\infty}$ [1]-algebras, we will suppress the adjective "flat" from now on. In this case the vanishing of the first Jacobiator implies that $\mu^{1}$ is a coboundary operator. We remark that an $L_{\infty}$ [1]-algebra structure on $V$ is equivalent to the more traditional notion of an $L_{\infty}$-algebra structure on $V[-1]$; see for instance [Markl et al. 2002].

Given an $L_{\infty}$-algebra structure $\left(\mu^{k}\right)_{k \geq 1}$ on $V$, there is a distinguished subset of $V_{1}$ that contains elements $v \in V_{1}$ satisfying the Maurer-Cartan equation (MC equation, for short)

$$
\sum_{k \geq 1} \frac{1}{k !} \mu^{k}(v \otimes \cdots \otimes v)=0
$$

This set is called the set of Maurer-Cartan elements (MC elements) of $V$.

Let $V$ be equipped with an $L_{\infty}$-algebra structure whose coboundary operator $\mu^{1}$ decomposes into $d+\delta$, with $d^{2}=0=\delta^{2}$ and $d \circ \delta+\delta \circ d=0$. That is, $(V, d, \delta)$ is a double complex. Then, under mild convergence assumptions, it is possible to construct an $L_{\infty}$-algebra structure on $H(V, d)$ that is isomorphic up to homotopy to the original $L_{\infty}$-algebra structure on $V$ [Gugenheim and Lambe 1989]. More concretely, one has to fix an embedding $i$ of $H(V, d)$ into $V$, a projection pr from $V$ to $H(V, d)$, and a homotopy operator $h$ (of degree -1 ) that satisfies

$$
d \circ h+h \circ d=\mathrm{id}_{V}-i \circ \mathrm{pr} .
$$


For simplicity, we will also impose the side conditions

$$
h \circ h=0, \quad \operatorname{pr} \circ h=0, \quad h \circ i=0 .
$$

Then explicit formulas for the structure maps of an $L_{\infty}$-algebra on $H(V, d)$ can be written down in terms of rooted planar trees; see [Schätz 2009a] for a review. We will explain the construction in more detail later for the examples that are relevant to our purpose.

One also obtains $L_{\infty}$ morphisms between $H(V, d)$ and $V$ that induce inverse maps on cohomology. Such $L_{\infty}$ morphisms are called $L_{\infty}$ quasi-isomorphisms.

Consider the differential graded algebra $\left(\Omega([0,1]), d_{D R}, \wedge\right)$ of smooth forms on the interval $I:=[0,1]$. The inclusions of a point $\{*\}$ for $s$ in $0 \leq s \leq 1$ induce a chain map ev $e_{s}:\left(\Omega(I), d_{D R}\right) \rightarrow(\mathbb{R}, 0)$ that is a morphisms of algebras. Given any $L_{\infty}$-algebra structure on $V$, there is a natural $L_{\infty}$-algebra structure on $V \otimes \Omega(I)$ defined by

$$
\begin{gathered}
\tilde{\mu}^{1}(v \otimes \alpha):=\mu^{1}(v) \otimes \alpha+(-1)^{|v|} v \otimes d_{D R} \alpha, \\
\tilde{\mu}^{k}\left(\left(v_{1} \otimes \alpha_{1}\right) \otimes \cdots \otimes\left(v_{k} \otimes \alpha_{k}\right)\right):=(-1)^{\#} \mu^{k}\left(v_{1} \otimes \cdots \otimes v_{k}\right) \otimes\left(\alpha_{1} \wedge \cdots \wedge \alpha_{k}\right)
\end{gathered}
$$

for $k \geq 2$. Here \# denotes the sign one picks up by assigning $(-1)^{\left|v_{i+1}\right|\left|\alpha_{i}\right|}$ to passing $\alpha_{i}$ from the left side of $v_{i+1}$ to the right side (and replacing $\alpha_{i+1}$ by $\alpha_{i} \wedge \alpha_{i+1}$ ).

Following [Markl et al. 2002], we call two $L_{\infty}$ morphisms $f$ and $g$ from $A$ to $B$ homotopic if there exists an $L_{\infty}$ morphism $F$ from $A$ to $B \otimes \Omega(I)$ such that

$$
\left(\mathrm{id} \otimes \mathrm{ev}_{0}\right) \circ F=f \quad \text { and } \quad\left(\mathrm{id} \otimes \mathrm{ev}_{1}\right) \circ F=g .
$$

This defines an equivalence relation on the set of $L_{\infty}$ morphisms from $A$ to $B$.

If $F$ is an $L_{\infty}$ morphism from $A$ to $B \otimes \Omega(I)$, then $f_{s}:=\mathrm{ev}_{s} \circ F$ is an $L_{\infty}$ morphism between $A$ and $B$ for any $s \in I$. Given an MC element $v$ in $A$, one obtains a one-parameter family

$$
w_{s}:=\sum_{k \geq 1} \frac{1}{n !}\left(f_{s}\right)_{k}(v \otimes \cdots \otimes v)
$$

of MC elements of $B$. Here $\left(f_{s}\right)_{k}$ denotes the $k$-th Taylor component of $f_{s}$.

In the main body of this paper, we are only interested in the case that $B$ is a differential graded Lie algebra (that is, only the first and second structure maps are nonvanishing). Denote the graded Lie bracket by $[\cdot, \cdot]$. Furthermore, assume that the differential $D$ is given by the adjoint action of a degree +1 element $\Gamma$ that satisfies $[\Gamma, \Gamma]=0$. The MC equation for an element $w$ of $(B, D=[\Gamma, \cdot],[\cdot, \cdot])$ reads

$$
[\Gamma+w, \Gamma+w]=0 .
$$


From the one-parameter family of $\mathrm{MC}$ elements $w_{s}$ in $B$, one obtains a oneparameter family of differential graded Lie algebras on $B$ by setting

$$
D_{s}(\cdot):=\left[\Gamma+w_{s}, \cdot\right]
$$

while leaving the bracket unchanged.

How are the differential graded Lie algebras $\left(B, D_{s},[\cdot, \cdot]\right)$ related for different values of $s \in I$ ? To answer this we first apply the $L_{\infty}$ morphism $F: A \rightarrow B \otimes \Omega(I)$ to $v$ and obtain an MC element $w(t)+u(t) d t$ in $B \otimes \Omega(I)$. It is straightforward to check that $w(s)=w_{s}$ for all $s \in I$. The MC equation in $B \otimes \Omega(I)$ splits up into

$$
[\Gamma+w(t), \Gamma+w(t)]=0 \quad \text { and } \quad \frac{d}{d t} w(t)=[u(t), \Gamma+w(t)] .
$$

The second equation implies that if the adjoint action of $u(t)$ on $B$ can be integrated to a one-parameter family of automorphisms $(U(t))_{t \in I}$, then $U(s)$ establishes an automorphism of $(B,[\cdot, \cdot])$ that maps $\Gamma+w(0)$ to $\Gamma+w(s)$ for any $s \in I$. Thus:

Lemma 2.1. Let $A$ and $(B,[\Gamma, \cdot],[\cdot, \cdot])$ be differential graded Lie algebras, let $v$ be an MC element in $A$, and let $F$ be an $L_{\infty}$ morphism from $A$ to $B \otimes \Omega(I)$ such that

$$
\sum_{k \geq 1} \frac{1}{k !} F_{k}(v \otimes \cdots \otimes v)
$$

is well-defined in $B \otimes \Omega(I)$; denote this element by $w(t)+u(t) d t$. Assume that the flow equation

$$
X(0)=b \quad \text { and }\left.\quad \frac{d}{d t}\right|_{t=s} X(t)=[u(s), X(s)] \quad \text { for } s \in I
$$

has a unique solution for arbitrary $b \in B$.

Then the one-parameter family $U(t)$ of automorphisms of $B$ that integrates the adjoint action by $u(t)$ maps $\Gamma+w(0)$ to $\Gamma+w(t)$. In particular, $U(s)$ is an isomorphism

$$
(B,[\Gamma+w(0), \cdot],[\cdot, \cdot]) \rightarrow(B,[\Gamma+w(s), \cdot],[\cdot, \cdot])
$$

of differential graded Lie algebras for arbitrary $s \in I$.

2b. Coisotropic submanifolds. We essentially follow [Weinstein 1988], omitting some details. Let $M$ be a smooth, finite-dimensional manifold. The bivector field $\Pi$ on $M$ is Poisson if the binary operation $\{\cdot, \cdot\}$ on $\mathscr{C}^{\infty}(M)$ given by $(f, g) \mapsto$ $\langle\Pi, d f \wedge d g\rangle$ satisfies the Jacobi identity; that is, if

$$
\{f,\{g, h\}\}=\{\{f, g\}, h\}+\{g,\{f, h\}\}
$$

holds for all smooth functions $f, g$ and $h$. Here, $\langle\cdot, \cdot\rangle$ denotes the natural pairing between $T M$ and $T^{*} M$. Alternatively, one can consider the graded algebra $\mathscr{V}(M)$ 
of multivector fields on $M$ equipped with the Schouten-Nijenhuis bracket $[\cdot, \cdot]_{\mathrm{SN}}$. A bivector field $\Pi$ is Poisson if and only if $[\Pi, \Pi]_{\mathrm{SN}}=0$.

Contraction associates to any Poisson bivector field $\Pi$ on $M$ a vector bundle morphism $\Pi^{\#}: T^{*} M \rightarrow T M$. Consider a submanifold $S$ of $M$. The annihilator $N^{*} S$ of $T S$ is a subbundle of $T^{*} M$. This subbundle fits into a short exact sequence $\left.0 \rightarrow N^{*} S \rightarrow T^{*} M\right|_{S} \rightarrow T^{*} S \rightarrow 0$ of vector bundles.

Definition 2.2. A submanifold $S$ of a smooth, finite-dimensional Poisson manifold $(M, \Pi)$ is called coisotropic if the restriction of $\Pi^{\#}$ to $N^{*} S$ has image in $T S$.

There is an equivalent characterization of coisotropic submanifolds: Define the vanishing ideal of $S$ by $\Phi_{S}:=\left\{f \in \mathscr{C}^{\infty}(M):\left.f\right|_{S}=0\right\}$. A submanifold $S$ is coisotropic if and only if $\Phi_{C}$ is a Lie subalgebra of $\left(\mathscr{C}^{\infty}(M),\{\cdot, \cdot\}\right)$.

2c. The BFV complex. The BFV complex was introduced by Batalin, Fradkin and Vilkovisky, with applications to physics in mind [Batalin and Fradkin 1983; Batalin and Vilkovisky 1977]. Later on, Stasheff [1997] gave an interpretation of the BFV complex in terms of homological algebra. The construction we present below is explained with more details in [Schätz 2009a]. It uses a globalization of the BFV complex for arbitrary coisotropic submanifolds found by Bordemann [2000] and Herbig [2006].

Let $S$ be a coisotropic submanifold of a smooth, finite-dimensional Poisson manifold $(M, \Pi)$. We outline the construction of a differential graded Poisson algebra, which we call a $B F V$ complex for $S$ in $(M, \Pi)$. The construction depends on the choice of three pieces of data: (1) an embedding of the normal bundle of $S$ into $M$ as a tubular neighborhood, (2) a connection on $N S$, and (3) a special smooth function, called the charge, on a smooth graded manifold.

Denote the normal bundle of $S$ inside $M$ by $E$. Consider the graded vector bundle $E^{*}[1] \oplus E[-1] \rightarrow S$ over $S$, and let $\mathscr{E}^{*}[1] \oplus \mathscr{E}[-1] \rightarrow E$ be the pullback of $E^{*}[1] \oplus E[-1] \rightarrow S$ along $E \rightarrow S$.

We define $\operatorname{BFV}(E)$ to be the space of smooth functions on the graded manifold that is represented by the graded vector bundle $\mathscr{E}^{*}[1] \oplus \mathscr{E}[-1]$ over $E$. In terms of sections, one has $\operatorname{BFV}(E)=\Gamma\left(\bigwedge \mathscr{E} \otimes \bigwedge_{\mathscr{E}} \mathscr{E}^{*}\right)$. This algebra carries a bigrading given by

$$
\operatorname{BFV}^{(p, q)}(E):=\Gamma\left(\bigwedge^{p} \mathscr{E} \otimes \bigwedge^{q} \mathscr{E}^{*}\right) .
$$

In physics terminology, $p$ and $q$ are referred to as the ghost degree and ghostmomentum degree, respectively. One defines

$$
\operatorname{BFV}^{k}(E):=\bigoplus_{p-q=k} \operatorname{BFV}^{(p, q)}(E),
$$

and calls $k$ the total degree (in physics terminology, this is the ghost number). 
The smooth graded manifold $\mathscr{E}^{*}[1] \oplus \mathscr{E}[-1]$ comes equipped with a Poisson bivector field $G$ given by the natural fiber-pairing between $\mathscr{E}$ and $\mathscr{E}^{*}$; that is, it is defined to be the natural contraction on $\Gamma(\mathscr{E}) \otimes \Gamma\left(\mathscr{E}^{*}\right)$ and is extended to a graded skew-symmetric biderivation of $\operatorname{BFV}(E)$.

Choice 1: Embedding. Fix an embedding $\psi: E \hookrightarrow M$ of the normal bundle of $S$ into $M$. Hence, the normal bundle $E$ inherits a Poisson bivector field which we also denote by $\Pi$. (Keep in mind that $\Pi$ depends on $\psi$.)

Choice 2: Connection. Next choose a connection on the vector bundle $E \rightarrow S$. This induces a connection on $\bigwedge E \otimes \wedge E^{*} \rightarrow S$ and, via pullback, one obtains a connection $\nabla$ on $\bigwedge \mathscr{E} \otimes \bigwedge_{\mathscr{E}}^{*} \rightarrow E$. We denote the corresponding horizontal lift of multivector fields by

$$
\iota_{\nabla}: \mathscr{V}(E) \rightarrow \mathscr{V}\left(\mathscr{E}^{*}[1] \otimes \mathscr{E}[-1]\right)
$$

It extends to an isomorphism

$$
\varphi: \mathscr{A}:=\mathscr{C}^{\infty}\left(T^{*}[1] E \oplus \mathscr{E}^{*}[1] \oplus \mathscr{E}[-1] \oplus \mathscr{E}[0] \oplus \mathscr{E}^{*}[2]\right) \rightarrow \mathscr{V}\left(\mathscr{E}^{*}[1] \oplus \mathscr{E}[-1]\right)
$$

of graded commutative unital associative algebras. Using $\varphi$, we lift $\Pi$ to a bivector field on $\mathscr{E}^{*}[1] \oplus \mathscr{E}[-1]$. Since in general $\varphi$ fails to be a morphism of Gerstenhaber algebras, $\varphi(\Pi)$ is not a Poisson bivector field. Similarly, the sum $G+\varphi(\Pi)$ fails to be a Poisson bivector field in general. However, the following proposition provides an appropriate correction term:

Proposition 2.3. Let $\mathscr{E}$ be a finite-rank vector bundle with connection $\nabla$ over a smooth, finite-dimensional manifold E. Consider the smooth graded manifold $\mathscr{E}^{*}[1] \oplus \mathscr{E}[-1] \rightarrow E$, and denote by $G$ the Poisson bivector field on it coming from the natural fiber pairing between $\mathscr{E}$ and $\mathscr{E} *$.

Then there is an $L_{\infty}$ quasi-isomorphism $\mathscr{L}_{\nabla}$ between the graded Lie algebra

$$
\left(\mathscr{V}(E)[1],[\cdot, \cdot]_{\mathrm{SN}}\right)
$$

and the differential graded Lie algebra

$$
\left(\mathscr{V}\left(\mathscr{E}^{*}[1] \oplus \mathscr{E}[-1]\right)[1],[G, \cdot]_{\mathrm{SN}},[\cdot, \cdot]_{\mathrm{SN}}\right) .
$$

A proof can be found in [Schätz 2009a]. Immediately, we have:

Corollary 2.4. Let $\mathscr{E} \rightarrow E$ be a finite-rank vector bundle with connection $\nabla$ over a smooth, finite-dimensional Poisson manifold $(E, \Pi)$. Consider the smooth graded manifold $\mathscr{E}^{*}[1] \oplus \mathscr{E}[-1] \rightarrow E$, and denote by $G$ the Poisson bivector field on it coming from the natural fiber pairing between $\mathscr{E}$ and $\mathscr{E}^{*}$.

Then, there is a Poisson bivector field $\hat{\Pi}$ on $\mathscr{E} *[1] \oplus \mathscr{E}[-1]$ such that

$$
\hat{\Pi}=G+\varphi(\Pi)+\Delta \text { for } \Delta \in \mathscr{V}^{(1,1)}\left(\mathscr{E}^{*}[1] \oplus \mathscr{E}[-1]\right) .
$$


For a proof, see again [Schätz 2009a].

We remark that $\mathscr{V}^{(1,1)}\left(\mathscr{E}^{*}[1] \oplus \mathscr{E}[-1]\right)$ is the ideal of $\mathscr{V}\left(\mathscr{E}^{*}[1] \oplus \mathscr{E}[-1]\right)$ that is generated by the multiderivations that map any tensor product of functions of total bidegree $(p, q)$ to a function of bidegree $(P, Q)$, where $P>p$ and $Q>q$. In general, let $\mathscr{V}^{(r, s)}\left(\mathscr{E}^{*}[1] \oplus \mathscr{E}[-1]\right)$ be the ideal generated by the multiderivations of $\mathscr{C}^{\infty}\left(\mathscr{E}^{*}[1] \oplus \mathscr{E}[-1]\right)$ with total ghost degree larger than or equal to $r$ and with total ghost-momentum degree larger than or equal to $s$, respectively.

The bivector field $\hat{\Pi}$ from Corollary 2.4 equips $\mathscr{E}^{*}[1] \oplus \mathscr{E}[-1]$ with the structure of a graded Poisson manifold. Consequently, $\operatorname{BFV}(E)$ inherits a graded Poisson bracket $[\cdot, \cdot]_{\mathrm{BFV}}$, called the BFV bracket. Keep in mind that the BFV bracket depends on the connection we have chosen on $E \rightarrow S$.

Choice 3: Charge. The last step in the construction of the BFV complex is to provide a special solution to the $\mathrm{MC}$ equation associated to $\left(\mathrm{BFV}(E),[\cdot, \cdot]_{\mathrm{BFV}}\right)$; that is, one constructs a degree +1 element $\Omega$ that satisfies

$$
[\Omega, \Omega]_{\mathrm{BFV}}=0 .
$$

Additionally, one requires that this element $\Omega$ contains the tautological section of $\mathscr{E} \rightarrow E$ as the lowest order term. To be more precise, recall that

$$
\operatorname{BFV}^{1}(E)=\bigoplus_{k \geq 0} \Gamma\left(\bigwedge^{k} \mathscr{E} \otimes \bigwedge^{k-1} \mathscr{E}^{*}\right) .
$$

Hence, any element of $\mathrm{BFV}^{1}(E)$ contains a (possibly zero) component in $\Gamma(\mathscr{E})$. One requires that the component of $\Omega$ in $\Gamma(\mathscr{E})$ be given by the tautological section of $\mathscr{E} \rightarrow E$. A MC element satisfying this requirement is called a BFV charge.

Proposition 2.5. Let $(E, \Pi)$ be a vector bundle equipped with a Poisson bivector field, and denote its zero section by $S$. Fix a connection on $E \rightarrow S$, and equip the ghost/ghost-momentum bundle $\mathscr{E}^{*}[1] \oplus \mathscr{E}[-1] \rightarrow E$ with the corresponding $B F V$ bracket $[\cdot, \cdot]_{\mathrm{BFV}}$.

(1) There is a degree +1 element $\Omega$ of $\operatorname{BFV}(E)$ whose component in $\Gamma(\mathscr{E})$ is given by the tautological section $\Omega_{0}$ and that satisfies

$$
[\Omega, \Omega]_{\mathrm{BFV}}=0
$$

if and only if $S$ is a coisotropic submanifold of $(E, \Pi)$.

(2) If $\Omega$ and $\Omega^{\prime}$ are two BFV charges, then there is an automorphism of the graded Poisson algebra $\left(\mathrm{BFV}(E),[\cdot, \cdot]_{\mathrm{BFV}}\right)$ that maps $\Omega$ to $\Omega^{\prime}$.

See [Stasheff 1997] for a proof.

Given a BFV charge $\Omega$, one can define a differential $D_{\mathrm{BFV}}(\cdot):=[\Omega, \cdot]_{\mathrm{BFV}}$, called $B F V$ differential. It is well known that the cohomology with respect to $D$ is 
isomorphic to the Lie algebroid cohomology of $S$ (as a coisotropic submanifold of $(E, \Pi))$.

By the second part of Proposition 2.5, different choices of the BFV charge lead to isomorphic differential graded Poisson algebra structures on $\operatorname{BFV}(E)$. In the next section, we will establish that different choices of connection on $E \rightarrow S$ lead to differential Poisson algebras that lie in the same isomorphism class. The dependence on the embedding of the normal bundle of $S$ is more subtle and will be clarified in Section 4.

\section{Choice of connection}

Consider a vector bundle $E$ equipped with a Poisson bivector field $\Pi$ such that its zero section $S$ is coisotropic. This section investigates how the differential graded Poisson algebra $\left(\mathrm{BFV}(E), D_{\mathrm{BFV}},[\cdot, \cdot]_{\mathrm{BFV}}\right)$ constructed in Section $2 \mathrm{c}$ depends on the choice of connection $\nabla$ on $E \rightarrow S$.

Recall a connection $\nabla$ on $E \rightarrow S$ was used to lift the Poisson bivector field $\Pi$ to a bivector field on $\mathscr{E}^{*}[1] \oplus \mathscr{E}[-1]$. The $L_{\infty}$ quasi-isomorphism between

$$
\left(\mathscr{V}(E)[1],[\cdot, \cdot]_{\mathrm{SN}}\right) \quad \text { and } \quad\left(\mathscr{V}\left(\mathscr{E}^{*}[1] \oplus \mathscr{E}[-1]\right)[1],[G, \cdot]_{\mathrm{SN}},[\cdot, \cdot]_{\mathrm{SN}}\right)
$$

in Proposition 2.3 depends on $\nabla$ too. Consequently, so does the graded Poisson bracket $[\cdot, \cdot]_{\mathrm{BFV}}$ on $\operatorname{BFV}(E)$.

Let $\nabla_{0}$ and $\nabla_{1}$ be two connections on a smooth finite-rank vector bundle $\mathscr{E} \rightarrow E$. By Proposition 2.3, we obtain two $L_{\infty}$ quasi-isomorphisms $\mathscr{L}_{\nabla_{0}}$ and $\mathscr{L}_{\nabla_{1}}$ from $\left(\mathscr{V}(E)[1],[\cdot, \cdot]_{\mathrm{SN}}\right)$ to $\left(\mathscr{V}\left(\mathscr{E}^{*}[1] \oplus \mathscr{E}[-1]\right)[1],[G, \cdot]_{\mathrm{SN}},[\cdot, \cdot]_{\mathrm{SN}}\right)$. Although the morphisms depend on the connections, this dependence is very well controlled:

Proposition 3.1. Let $\mathscr{E}$ be a smooth finite-rank vector bundle over a smooth, finitedimensional manifold $E$ equipped with two connections $\nabla_{0}$ and $\nabla_{1}$. Denote by $\mathscr{L}_{0}$ and $\mathscr{L}_{1}$ the associated $L_{\infty}$ quasi-isomorphisms from Proposition 2.3 between $\left(\mathscr{V}(E)[1],[\cdot, \cdot]_{\mathrm{SN}}\right)$ and $\left(\mathscr{V}(\mathscr{E} *[1] \oplus \mathscr{E}[-1]),[G, \cdot]_{\mathrm{SN}},[\cdot, \cdot]_{\mathrm{SN}}\right)$, respectively.

Then there is an $L_{\infty}$ quasi-isomorphism

$\hat{\mathscr{L}}:\left(\mathscr{V}(E)[1],[\cdot, \cdot]_{\mathrm{SN}}\right) \rightarrow\left(\mathscr{V}\left(\mathscr{E}^{*}[1] \oplus \mathscr{E}[-1]\right) \otimes \Omega(I),[G, \cdot]_{\mathrm{SN}}+d_{D R},[\cdot, \cdot]_{\mathrm{SN}}\right)$ such that $\left(\mathrm{id} \otimes \mathrm{ev}_{0}\right) \circ \hat{\mathscr{L}}=\mathscr{L}_{0}$ and $\left(\mathrm{id} \otimes \mathrm{ev}_{1}\right) \circ \hat{\mathscr{L}}=\mathscr{L}_{1}$.

Proof. Given two connections $\nabla_{0}$ and $\nabla_{1}$, one can define a family of connections $\nabla_{s}:=\nabla_{0}+s\left(\nabla_{1}-\nabla_{0}\right)$ parametrized by the closed unit interval $I$. Consequently we obtain a one-parameter family of isomorphisms of graded algebras

$$
\varphi_{s}: \mathscr{A}:=\mathscr{C}^{\infty}\left(T^{*}[1] E \oplus \mathscr{\mathscr { E }}^{*}[1] \oplus \mathscr{E}[-1] \oplus \mathscr{E}[0] \oplus \mathscr{E}^{*}[2]\right) \stackrel{\cong}{\longrightarrow} \mathscr{V}\left(\mathscr{E}^{*}[1] \oplus \mathscr{E}[-1]\right),
$$

extending the horizontal lift with respect to the connection $\nabla_{s} \oplus \nabla_{s}^{*}$. Via this identification, $\mathscr{A}$ inherits a one-parameter family of Gerstenhaber brackets, which 
we denote by $[\cdot, \cdot]_{s}$, and a differential $\widetilde{Q}$, which can be checked to be independent of $s$ in local coordinates.

For arbitrary $s \in I$, these structures fit into the commutative diagram

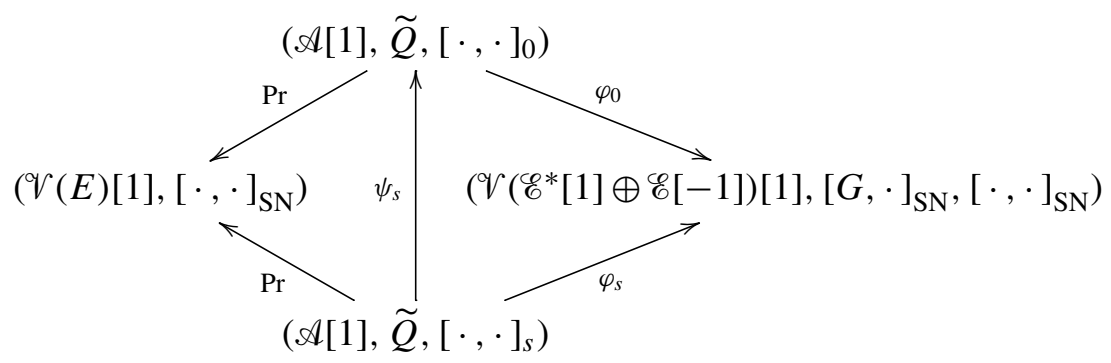

where $\psi_{s}:=\varphi_{0}^{-1} \circ \varphi_{s}$ is a morphism of differential graded algebras and of Gerstenhaber algebras. Pr denotes the natural projection.

It is straightforward to show that the cohomology of $(\mathscr{A}, \widetilde{Q})$ is $\mathscr{V}(E)$, and that the induced $L_{\infty}$ algebra coincides with $(\mathscr{V}(E)[1],[\cdot, \cdot])$; see [Schätz 2009a, proof of Proposition 1]. Hence we obtain a one-parameter family of $L_{\infty}$ quasi-isomorphisms $\mathscr{F}_{s}:\left(\mathscr{V}(E)[1],[\cdot, \cdot]_{\mathrm{SN}}\right) \rightarrow\left(\mathscr{A}[1], \widetilde{Q},[\cdot, \cdot]_{s}\right)$. Composition with $\psi_{s}$ yields a one-parameter family of $L_{\infty}$ quasi-isomorphisms

$$
\mathscr{K}_{s}:\left(\mathscr{V}(E)[1],[\cdot, \cdot]_{\mathrm{SN}}\right) \rightarrow\left(\mathscr{A}[1], \widetilde{Q},[\cdot, \cdot]_{0}\right) .
$$

We remark that the composition of $\mathscr{S}_{s}$ with $\varphi_{s}$ yields the $L_{\infty}$ quasi-isomorphism $\mathscr{L}_{s}$ between $\left(\mathscr{V}(E),[\cdot, \cdot]_{\mathrm{SN}}\right)$ and $\left(\mathscr{V}\left(\mathscr{E}^{*}[1] \oplus E[-1]\right),[G, \cdot]_{\mathrm{SN}},[\cdot, \cdot]_{\mathrm{SN}}\right)$ associated to the connection $\nabla_{s}$ from Proposition 2.3. Consequently, $\mathscr{L}_{0}$ and $\mathscr{L}_{1}$ are the compositions of $\mathscr{K}_{0}$ and $\mathscr{K}_{1}$, respectively, with $\varphi_{0}$.

Next consider the differential graded Lie algebra

$$
\left(\mathscr{A}[1] \otimes \Omega(I), \widetilde{Q}+d_{D R},[\cdot, \cdot]_{0}\right) .
$$

To prove Proposition 2.3, a homotopy $\widetilde{H}$ for $\widetilde{Q}$ was constructed in [Schätz 2009a] such that $\widetilde{Q} \circ \widetilde{H}+\widetilde{H} \circ \widetilde{Q}=\mathrm{id}-\iota \circ \operatorname{Pr}$. Here $\iota$ denotes the natural inclusion $\mathscr{V}(E) \hookrightarrow \mathscr{A}$. One defines a one-parameter family of homotopies $\widetilde{H}_{s}:=\psi_{s} \circ \widetilde{H} \circ \psi_{s}^{-1}$ and checks that $\widetilde{Q} \circ \widetilde{H}_{s}+\widetilde{H}_{s} \circ \widetilde{Q}=\mathrm{id}-\psi_{s} \circ \iota \circ \operatorname{Pr}$.

We define $\widehat{\operatorname{Pr}}: \mathscr{A} \otimes \Omega(I) \rightarrow \mathscr{V}(E) \otimes \Omega(I)$ to be $\operatorname{Pr} \otimes$ id, and $\hat{\imath}: \mathscr{V}(E) \otimes \Omega(I) \rightarrow$ $\mathscr{A} \otimes \Omega(I)$ to be $\hat{\imath}:=\left(\psi_{s} \circ \iota\right) \otimes$ id. Clearly $\widehat{\operatorname{Pr}} \circ \hat{\imath}=\mathrm{id}$ and $\widetilde{H}_{s}$ provides a homotopy between id and $\hat{\imath} \circ \widehat{\operatorname{Pr}}$. Moreover, the side conditions $\widetilde{H}_{s} \circ \widetilde{H}_{s}=0$, $\widehat{\operatorname{Pr}} \circ \widetilde{H}_{s}=0$ and $\widetilde{H}_{s} \circ \hat{\imath}=0$ are still satisfied. We summarize the situation in the diagram

$$
(\mathscr{V}(E) \otimes \Omega(I), 0) \underset{\widehat{\operatorname{Pr}}}{\stackrel{\hat{\iota}_{s}}{\rightleftarrows}}(\mathscr{A} \otimes \Omega(I), \widetilde{Q}), \widetilde{H}_{s} .
$$


Following Section 2a, these data can be used to perform homological transfer. The input consists of the differential graded Lie algebra

$$
\left(\mathscr{A}[1] \otimes \Omega(I), \widetilde{Q}+d_{D R},[\cdot, \cdot]_{0}\right) .
$$

To construct the induced structure maps, one has to consider oriented rooted trees with bivalent and trivalent interior vertices. The leaves (the exterior vertices with the root excluded) are decorated by $\hat{\imath}$, the root by $\widehat{P r}$, the interior bivalent vertices by $d_{D R}$, the interior trivalent vertices by $[\cdot, \cdot]_{0}$, and the interior edges (that is, the edges not connected to any exterior vertices) by $-\widetilde{H}_{s}$. One then composes these maps in the order given by the orientation towards the root. The associated $L_{\infty}$ quasi-isomorphism is constructed in the same manner; however, the root is not decorated by $\widehat{\operatorname{Pr}}$ but by $-\widetilde{H}_{s}$ instead.

Recall that $\mathscr{V}^{(r, s)}\left(\mathscr{E}^{*}[1] \oplus \mathscr{E}[-1]\right)$ is the ideal generated by the multiderivations of $\mathscr{C}^{\infty}\left(\mathscr{E}^{*}[1] \oplus \mathscr{E}[-1]\right)$ with total ghost degree larger than or equal to $r$, and total ghost-momentum degree larger than or equal to $s$, respectively. One can check inductively that trees decorated with $e$ copies of $-\widetilde{H}_{s}$ increase the filtration index by $(e, e)$. Also, trees containing more than one interior bivalent vertex do not contribute since $d_{D R}$ increases the form degree by 1 . These facts imply that the induced structure is given by $\left(\mathscr{V}(E)[1] \otimes \Omega(I), d_{D R},[\cdot, \cdot]_{\mathrm{SN}}\right)$, and that there is an $L_{\infty}$ quasi-isomorphism

$$
\left(\mathscr{V}(E)[1] \otimes \Omega(I), d_{D R},[\cdot, \cdot]_{\mathrm{SN}}\right) \rightarrow\left(\mathscr{A}[1] \otimes \Omega(I), \widetilde{Q}+d_{D R},[\cdot, \cdot]_{0}\right) .
$$

We define

$$
\widetilde{\mathscr{K}}:\left(\mathscr{V}(E)[1],[\cdot, \cdot]_{\mathrm{SN}}\right) \rightarrow\left(\mathscr{V}\left(\mathscr{A}[1] \otimes \Omega(I), \widetilde{Q}+d_{D R},[\cdot, \cdot]_{\mathrm{SN}}\right)\right.
$$

to be the composition of this $L_{\infty}$ quasi-isomorphism and the obvious $L_{\infty}$ quasiisomorphism $\left(\mathscr{V}(E)[1],[\cdot, \cdot]_{\mathrm{SN}}\right) \hookrightarrow\left(\mathscr{V}(E)[1] \otimes \Omega(I), d_{D R},[\cdot, \cdot]_{\mathrm{SN}}\right)$.

The composition of $\hat{\mathscr{K}}$ with id $\otimes \mathrm{ev}_{s}: \mathscr{A} \otimes \Omega(I) \rightarrow \mathscr{A}$ can be computed as follows. First, only trees without any bivalent interior edges contribute since all elements of form degree 1 vanish under id $\otimes \mathrm{ev}_{s}$. Using the identities

$$
\psi_{s}^{-1}\left(\left[\psi_{s}(\cdot), \psi_{s}(\cdot)\right]_{0}\right)=[\cdot, \cdot]_{s}, \quad \tilde{H}_{s}=\psi_{s} \circ \tilde{H} \circ \psi_{s}^{-1}, \quad \hat{\imath}=\psi_{s} \circ \iota,
$$

it is straightforward to show that $\left(\mathrm{id} \otimes \mathrm{ev}_{s}\right) \circ \hat{\mathscr{K}}=\psi_{s} \circ \mathscr{K}_{s}$. Hence

$$
\varphi_{0} \circ\left(\mathrm{id} \otimes \mathrm{ev}_{s}\right) \circ \hat{\mathscr{K}}=\varphi_{s} \circ \mathscr{K}_{s}=\mathscr{L}_{s} .
$$

Finally, we define the $L_{\infty}$ quasi-isomorphism $\hat{\mathscr{L}}$ between $\left(\mathscr{V}(E)[1],[\cdot, \cdot]_{\mathrm{SN}}\right)$ and

$$
\left(\mathscr{V}\left(\mathscr{E}^{*}[1] \oplus \mathscr{E}[-1]\right)[1] \otimes \Omega(I),[G, \cdot]_{\mathrm{SN}}+d_{D R},[\cdot, \cdot]_{\mathrm{SN}}\right)
$$

to be $\left(\varphi_{0} \otimes \mathrm{id}\right) \circ \hat{\mathscr{K}}$. By construction, $\left(\mathrm{id} \otimes \mathrm{ev}_{0}\right) \circ \hat{\mathscr{L}}=\mathscr{L}_{0}$ and $\left(\mathrm{id} \otimes \mathrm{ev}_{1}\right) \circ \hat{\mathscr{L}}=\mathscr{L}_{1}$ are satisfied. 
Propositions 2.3 and 3.1 seem to permit higher analogues where one incorporates the differential graded algebra of differential forms on the $n$-simplex $\Omega\left(\triangle^{n}\right)$ instead of just $\Omega(\{*\})=\mathbb{R}$, as in Proposition 2.3, or $\Omega(I)$, as in Proposition 3.1; see [Costello 2007], where this idea was worked out in the context of the BV formalism.

Corollary 3.2. Let $\mathscr{E}$ be a finite-rank vector bundle over a smooth, finite-dimensional Poisson manifold $(E, \Pi)$. Let $\nabla_{0}$ and $\nabla_{1}$ be two connections on $\mathscr{E} \rightarrow E$. Denote by $\mathscr{L}_{0}$ and $\mathscr{L}_{1}$ the associated $L_{\infty}$ quasi-isomorphisms between

$$
\left(\mathscr{V}(E)[1],[\cdot, \cdot]_{\mathrm{SN}}\right) \quad \text { and } \quad\left(\mathscr{V}\left(\mathscr{E}^{*}[1] \oplus \mathscr{E}[-1]\right)[1],[G, \cdot]_{\mathrm{SN}},[\cdot, \cdot]_{\mathrm{SN}}\right)
$$

from Proposition 2.3, respectively. Applying these $L_{\infty}$ quasi-isomorphisms to $\Pi$ yields two MC elements $\widetilde{\Pi}_{0}$ and $\widetilde{\Pi}_{1}$ of

$$
\left(\mathscr{V}\left(\mathscr{E}^{*}[1] \oplus \mathscr{E}[-1]\right)[1],[G, \cdot]_{\mathrm{SN}},[\cdot, \cdot]_{\mathrm{SN}}\right) .
$$

Hence

$$
\hat{\Pi}_{0}:=G+\widetilde{\Pi}_{0} \quad \text { and } \quad \hat{\Pi}_{1}:=G+\widetilde{\Pi}_{1}
$$

are MC elements of $\left(\mathscr{V}\left(\mathscr{E}^{*}[1] \oplus \mathscr{E}[-1]\right)[1],[\cdot, \cdot]_{\mathrm{SN}}\right)$, that is, Poisson bivector fields on $\mathscr{E} *[1] \oplus \mathscr{E}[-1]$.

There is a diffeomorphism of the smooth graded manifold $\mathscr{E}^{*}[1] \oplus \mathscr{E}[1]$ such that the induced automorphism of $\mathscr{V}\left(\mathscr{E}^{*}[1] \oplus \mathscr{E}[-1]\right)$ maps $\hat{\Pi}_{0}$ to $\hat{\Pi}_{1}$. This diffeomorphism induces a diffeomorphism of the base E that coincides with the identity.

Proof. Apply the $L_{\infty}$ quasi-isomorphism $\hat{\mathscr{L}}$ from Proposition 3.1 to $\Pi$, and add $G$ to obtain an MC element $\hat{\Pi}+\hat{Z} d t$ of $\left(\mathscr{V}\left(\mathscr{E}^{*}[1] \oplus \mathscr{E}[-1]\right)[1] \otimes \Omega(I), d_{D R},[\cdot, \cdot]_{\mathrm{SN}}\right)$. Let $\mathscr{L}_{s}$ denote the $L_{\infty}$ quasi-isomorphism from Proposition 2.3 constructed with the help of the connection $\nabla_{0}+s\left(\nabla_{1}-\nabla_{0}\right)$. Recall that $\left(\mathrm{id} \otimes \mathrm{ev}_{s}\right) \circ \hat{\mathscr{L}}=\mathscr{L}_{s}$ holds for all $s \in I$.

We set $\hat{\Pi}_{s}:=\left(\operatorname{id} \otimes \mathrm{ev}_{s}\right)(\hat{\Pi})$ and $\hat{Z}_{s}:=\left(\mathrm{id} \otimes \mathrm{ev}_{s}\right)(\hat{Z})$. Proposition 3.1 implies that this definition of $\hat{\Pi}_{s}$ is compatible with the $\hat{\Pi}_{0}$ and $\hat{\Pi}_{1}$ defined in our corollary.

We want to apply Lemma 2.1 to

$$
A:=\left(\mathscr{V}(E)[1],[\cdot, \cdot]_{\mathrm{SN}}\right), \quad B:=\left(\mathscr{V}\left(\mathscr{E}^{*}[1] \oplus \mathscr{E}[-1]\right)[1],[G, \cdot]_{\mathrm{SN}},[\cdot, \cdot]_{\mathrm{SN}}\right)
$$

and $F:=\hat{\mathscr{L}}$. To do so, it remains to show that the flow of $\hat{Z}_{s}$ is globally well-defined for $s \in[0,1]$. Recall that $\hat{Z}$ is the one-form part of the MC element constructed from the Poisson bivector field $\Pi$ on $E$ with the help of the $L_{\infty}$ quasi-isomorphism $\hat{\mathscr{L}}:\left(\mathscr{V}(E)[1],[\cdot, \cdot]_{\mathrm{SN}}\right) \rightarrow\left(\mathscr{V}\left(\mathscr{E}^{*}[1] \oplus \mathscr{E}[-1]\right) \otimes \Omega(I),[G, \cdot]_{\mathrm{SN}}+d_{D R},[\cdot, \cdot]_{\mathrm{SN}}\right)$. Only trees with exactly one bivalent interior vertex give nonzero contributions because the form degree must be one. Therefore, there is at least one homotopy in the diagram, and by the degree estimate in the proof of Proposition 3.1, this implies that $\hat{Z}$ is contained in $\mathscr{V}^{(1,1)}\left(\mathscr{E}^{*}[1] \oplus \mathscr{E}[-1]\right) \otimes \Omega(I)$. Hence the derivation $[\hat{Z}, \cdot]_{\mathrm{SN}}$ is 
nilpotent and can be integrated. Furthermore, the degree estimate directly implies the last claim of our corollary.

The following is an immediate consequence of the previous corollary:

Corollary 3.3. Let $(E, \Pi)$ be a vector bundle $E \rightarrow S$ equipped with a Poisson structure $\Pi$ such that $S$ is a coisotropic submanifold. Fix two connections $\nabla_{0}$ and $\nabla_{1}$ on $E \rightarrow S$, and denote the corresponding graded Poisson brackets on $\mathrm{BFV}(E)$ by $[\cdot, \cdot]_{\mathrm{BFV}}^{0}$ and $[\cdot, \cdot]_{\mathrm{BFV}}^{1}$, respectively.

There is an isomorphism

$$
\left(\mathrm{BFV}(E),[\cdot, \cdot]_{\mathrm{BFV}}^{0}\right) \stackrel{\cong}{\longrightarrow}\left(\mathrm{BFV}(E),[\cdot, \cdot]_{\mathrm{BFV}}^{0}\right)
$$

of graded Poisson algebras. The induced automorphism of $\mathscr{C}^{\infty}(E)$ coincides with the identity.

Combining Proposition 2.5 and Corollary 3.3, we obtain a theorem:

Theorem 3.4. Let $E$ be a vector bundle equipped with a Poisson bivector $\Pi$ such that the zero section $S$ is a coisotropic submanifold. Recall that the pullback of $E \rightarrow S$ by $E \rightarrow S$ is denoted by $\mathscr{E} \rightarrow E$, and that

$$
\operatorname{BFV}(E):=\mathscr{C}^{\infty}\left(\mathscr{E}^{*}[1] \oplus \mathscr{E}[-1]\right)=\Gamma\left(\bigwedge_{\mathscr{E}} \otimes \bigwedge^{\mathscr{E}^{*}}\right) .
$$

Different choices of a connection $\nabla$ on $E \rightarrow S$ and of a degree +1 element $\Omega$ of $\left(\operatorname{BFV}(S),[\cdot, \cdot]_{\mathrm{BFV}}\right)$ for which

(1) the lowest-order term of $\Omega$ is given by the tautological section $\Omega_{0}$ of $\mathscr{E} \rightarrow E$ and

(2) $[\Omega, \Omega]_{\mathrm{BFV}}^{\nabla}=0$

lead to isomorphic differential graded Poisson algebras

$$
\left(\mathrm{BFV}(E),[\Omega, \cdot]_{\mathrm{BFV}}^{\nabla},[\cdot, \cdot]_{\mathrm{BFV}}^{\nabla}\right) .
$$

Proof. Pick two connections $\nabla_{0}$ and $\nabla_{1}$ on $E \rightarrow S$, and consider the associated graded Poisson algebras $\left(\mathrm{BFV}(E),[\cdot, \cdot]_{\mathrm{BFV}}^{0}\right)$ and $\left(\mathrm{BFV}(E),[\cdot, \cdot]_{\mathrm{BFV}}^{1}\right)$, respectively. By Corollary 3.3, there is an isomorphism

$$
\gamma:\left(\operatorname{BFV}(E),[\cdot, \cdot]_{\mathrm{BFV}}^{0}\right) \stackrel{\cong}{\longrightarrow}\left(\mathrm{BFV}(E),[\cdot, \cdot]_{\mathrm{BFV}}^{1}\right)
$$

of graded Poisson algebras. Moreover, the induced automorphism of $\mathscr{C}^{\infty}(E)$ is the identity. Assume that $\Omega$ and $\widetilde{\Omega}$ are two BFV charges of $\left(\operatorname{BFV}(E),[\cdot, \cdot]_{\mathrm{BFV}}^{0}\right)$ and $\left.\operatorname{BFV}(E),[\cdot, \cdot]_{\mathrm{BFV}}^{1}\right)$, respectively. Applying the automorphism $\gamma$ to $\Omega$ yields another element of $\left(\operatorname{BFV}(E),[\cdot, \cdot]_{\mathrm{BFV}}^{1}\right)$, which can be checked to be a BFV charge 
again. By Proposition 2.5, this implies that there is an inner automorphism $\beta$ of $\left.\operatorname{BFV}(E),[\cdot, \cdot]_{\mathrm{BFV}}^{1}\right)$ that maps $\gamma(\Omega)$ to $\widetilde{\Omega}$. Hence

$$
\beta \circ \gamma:\left(\operatorname{BFV}(E),[\cdot, \cdot]_{\mathrm{BFV}}^{0}\right) \stackrel{\cong}{\longrightarrow}\left(\mathrm{BFV}(E),[\cdot, \cdot]_{\mathrm{BFV}}^{1}\right)
$$

is an isomorphism of graded Poisson algebras that maps $\Omega$ to $\widetilde{\Omega}$.

\section{Choice of tubular neighborhood}

Suppose $S$ is a coisotropic submanifold of a smooth, finite-dimensional Poisson manifold $(M, \Pi)$. Throughout this section, $E$ denotes the normal bundle of $S$ inside $M$. As explained in Section 2c, the first step in the construction of the BFV complex for $S$ inside $(M, \Pi)$ is the choice of an embedding $\psi: E \hookrightarrow M$. Such an embedding equips $E$ with a Poisson bivector field $\Pi_{\psi}$, which is used to construct the BFV bracket on the ghost/ghost-momentum bundle; see Section 2c.

First, we consider the case where the embedding is changed by composition with a linear automorphism of the normal bundle $E$ :

Lemma 4.1. For some choice of tubular neighborhood $\psi: E \hookrightarrow M$, let

$$
\left(\mathrm{BFV}(E),[\Omega, \cdot]_{\mathrm{BFV}},[\cdot, \cdot]_{\mathrm{BFV}}\right)
$$

be the corresponding BFV complex; let

$$
\left(\mathrm{BFV}(E),\left[\Omega^{g}, \cdot\right]_{\mathrm{BFV}}^{g},[\cdot, \cdot]_{\mathrm{BFV}}^{g}\right)
$$

be a BFV complex corresponding to the embedding $\psi \circ g: E \hookrightarrow M$, where $g: E \rightarrow E$ is a vector-bundle isomorphism covering the identity.

Then there is an isomorphism of graded Poisson algebras

$$
\left(\operatorname{BFV}(E),[\cdot, \cdot]_{\mathrm{BFV}}\right) \rightarrow\left(\operatorname{BFV}(E),[\cdot, \cdot]_{\mathrm{BFV}}^{g}\right)
$$

that maps $\Omega$ to $\Omega^{g}$.

Proof. Let $\Pi$ and $\Pi^{g}$ be the Poisson bivector fields on $E$ obtained from $\psi: E \hookrightarrow M$ and $\psi \circ g: E \hookrightarrow M$, respectively. Clearly $\Pi^{g}=(g)_{*}(\Pi)$.

Choose some connection $\nabla$ of $E$, which is used to construct the $L_{\infty}$ quasiisomorphism

$$
\mathscr{L}:\left(\mathscr{V}(E)[1],[\cdot, \cdot]_{\mathrm{SN}}\right) \rightarrow\left(\mathscr{V}\left(\mathscr{E}^{*}[1] \oplus \mathscr{E}[-1]\right)[1],[\cdot, \cdot]_{\mathrm{SN}},[G, \cdot]_{\mathrm{SN}}\right)
$$

Plugging in $\Pi$ results in the BFV bracket $[\cdot, \cdot]_{\mathrm{BFV}}$. On the other hand, we can use $\nabla^{g}:=\left(g^{-1}\right)^{*} \nabla$ to construct another $L_{\infty}$ quasi-isomorphism $\mathscr{L}^{g}$. Plugging in $\Pi^{g}$ results in another $\mathrm{BFV}$ bracket $[\cdot, \cdot]_{\mathrm{BFV}}^{g}$. 
We claim that $[\cdot, \cdot]_{\mathrm{BFV}}$ and $[\cdot, \cdot]_{\mathrm{BFV}}^{g}$ are isomorphic graded Poisson brackets. First, observe that the isomorphism $g: E \rightarrow E$ lifts to a vector-bundle isomorphism

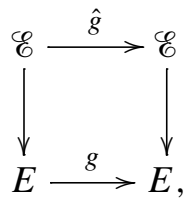

such that the tautological section gets mapped to itself under $(\hat{g})^{*}$. We denote the induced automorphism of $E^{*}[1] \oplus E[-1]$ by $\hat{g}$ as well.

By naturality of the pullback of connections, we obtain the commutative diagram

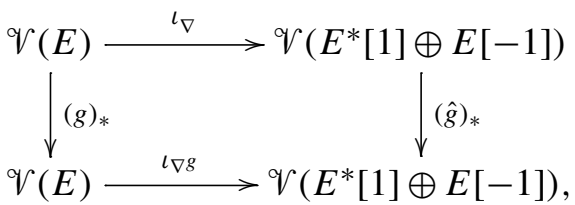

where $\iota_{\nabla}$ and $\iota_{\nabla^{g}}$ are the horizontal lifts induced by $\nabla$ and $\nabla^{g}$, respectively. Using this, together with the explicit description of the $L_{\infty}$ quasi-isomorphism $\mathscr{L}$ from Proposition 2.3 (contained in [Schätz 2009a] or in the proof of Proposition 3.1), one concludes that

$$
\left(\mathscr{L}^{g}\right)_{k}=(\hat{g})_{*} \circ(\mathscr{L})_{k} \circ\left((g)_{*}^{-1} \otimes \cdots \otimes(g)_{*}^{-1}\right) .
$$

Here $(\mathscr{L})_{k}$ denotes the $k$-th structure map of the $L_{\infty}$ quasi-isomorphism $\mathscr{L}$.

This immediately implies that $\hat{g}$ induces an isomorphism between $[\cdot, \cdot]_{\mathrm{BFV}}$ and $[\cdot, \cdot]_{\mathrm{BFV}}^{g}$. Since $\hat{g}$ maps the tautological section to itself, it maps any BFV charge to another one.

Finally, Theorem 3.4 implies the statement of Lemma 4.1.

A different choice of embedding can cause drastic changes in the associated BFV complexes. Consider $S=\{0\}$ inside $M=\mathbb{R}^{2}$ equipped with the smooth Poisson bivector field

$$
\Pi(x, y):= \begin{cases}0 & \text { for } x^{2}+y^{2} \leq 4, \\ \exp \left(-\frac{1}{x^{2}+y^{2}-4}\right) \frac{\partial}{\partial x} \wedge \frac{\partial}{\partial y} & \text { for } x^{2}+y^{2} \geq 4 .\end{cases}
$$

Let $\psi_{0}$ be the embedding of $E \cong \mathbb{R}^{2}$ into $\mathbb{R}^{2}$ given by the identity, and let $\psi_{1}$ be the embedding given by

$$
(x, y) \mapsto \frac{1}{\sqrt{1+x^{2}+y^{2}}}(x, y) .
$$

The image of $\psi_{1}$ is contained in the disk of radius 1 . Hence $\Pi_{\psi_{1}}$ vanishes identically whereas $\Pi_{\psi_{0}}$ does not. 
The ghost/ghost-momentum bundle $\mathscr{E}^{*}[1] \oplus \mathscr{E}[-1]$ is of the very simple form

$$
\mathbb{R}^{2} \times\left(\left(\mathbb{R}^{2}\right)^{*}[1] \oplus \mathbb{R}^{2}[-1]\right) \rightarrow \mathbb{R}^{2}
$$

Denote the Poisson bivector field coming from the natural pairing between $\left(\mathbb{R}^{2}\right)^{*}[1]$ and $\mathbb{R}^{2}[-1]$ by $G$. We choose the standard flat connection on the bundle $\mathbb{R}^{2} \rightarrow 0$. Then the Poisson bivector fields for the BFV brackets $[\cdot, \cdot]_{\mathrm{BFV}}^{0}$ and $[\cdot, \cdot]_{\mathrm{BFV}}^{1}$ are simply given by the sums $G+\Pi_{\psi_{0}}$ and $G+\Pi_{\psi_{1}}$, respectively.

Any isomorphism of graded Poisson algebras between $\left(\operatorname{BFV}(E),[\cdot, \cdot]_{\mathrm{BFV}}^{0}\right)$ and $\left(\operatorname{BFV}(E),[\cdot, \cdot]_{\mathrm{BFV}}^{1}\right)$ yields an induced isomorphism of Poisson algebras between $\left(\mathscr{C}^{\infty}\left(\mathbb{R}^{2}\right),\{\cdot, \cdot\}_{\Pi_{\psi_{0}}}\right)$ and $\left(\mathscr{C}^{\infty}\left(\mathbb{R}^{2}\right),\{\cdot, \cdot\}_{\Pi_{\psi_{0}}}\right)$. Since $\Pi_{\psi_{1}}$ vanishes, the induced automorphism would have to map something nonvanishing to 0 , which is a contradiction. Hence there is no isomorphism of graded Poisson algebras between $\left(\mathrm{BFV}(E),[\cdot, \cdot]_{\mathrm{BFV}}^{0}\right)$ and $\left(\mathrm{BFV}(E),[\cdot, \cdot]_{\mathrm{BFV}}^{1}\right)$.

Although different choices of embeddings can lead to differential graded Poisson algebras that are not isomorphic, it is always possible to find appropriate restrictions of the BFV complexes such that the corresponding differential graded Poisson algebras are isomorphic.

Definition 4.2. Let $E$ be a finite-rank vector bundle over a smooth manifold $S$. Assume $E$ is equipped with a Poisson bivector field $\Pi$ such that $S$ is a coisotropic submanifold of $E$. Let $\left(\operatorname{BFV}(E), D_{\mathrm{BFV}},[\cdot, \cdot]_{\mathrm{BFV}}\right)$ be a $\mathrm{BFV}$ complex for $S$ in $(E, \Pi)$, and $U$ an open neighborhood of $S$ inside $E$. The restriction of the BFV complex on $U$ is the differential graded Poisson algebra

$$
\left(\mathrm{BFV}^{U}(E), D_{\mathrm{BFV}}^{U}(\cdot)=\left[\Omega^{U}, \cdot\right]_{\mathrm{BFV}}^{U},[\cdot, \cdot]_{\mathrm{BFV}}^{U}\right)
$$

given by the following data:

(a) $\operatorname{BFV}^{U}(E)$ is the space of smooth functions on the graded vector bundle $(\mathscr{E} *[1]$ $\oplus \mathscr{E}[-1])\left.\right|_{U}$ fitting into the Cartesian square

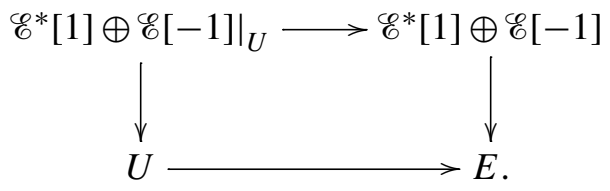

(b) $\operatorname{BFV}^{U}(E)$ inherits a graded Poisson bracket $[\cdot, \cdot]_{\mathrm{BFV}}^{U}$ from $\mathrm{BFV}(E)$, obtained by restricting the Poisson bivector field corresponding to $[\cdot, \cdot]_{\mathrm{BFV}}$ to the graded submanifold $\left.\left(\mathscr{E}^{*}[1] \oplus \mathscr{E}[-1]\right)\right|_{U}$ of $\mathscr{C}^{*}[1] \oplus \mathscr{E}[-1]$.

(c) An element $\Omega^{U}$ of $\mathrm{BFV}^{U}(E)$ is called a restricted $B F V$ charge if it is of degree +1 , if $\left[\Omega^{U}, \Omega^{U}\right]_{\mathrm{BFV}}^{U}=0$, and if the component of $\Omega^{U}$ in $\Gamma\left(\left.\mathscr{E}\right|_{U}\right)$ is equal to the restriction of the tautological section $\Omega_{0} \in \Gamma(\mathscr{E})$ to $U$. 
Proposition 4.3. Let $S$ be a coisotropic submanifold of a smooth, finite-dimensional Poisson manifold $(M, \Pi)$. Denote the normal bundle of $S$ by $E$ and fix a connection $\nabla$ on $E$. Let $\psi_{0}$ and $\psi_{1}$ be two embeddings of $E$ into $M$ as tubular neighborhoods of $S$.

Using these data, one builds two graded Poisson algebra structures on $\mathrm{BFV}(E)$ following Section 2c (in particular, one applies Proposition 2.3). Denote the two corresponding graded Poisson brackets by $[\cdot, \cdot]_{\mathrm{BFV}}^{0}$ and $[\cdot, \cdot]_{\mathrm{BFV}}^{1}$, respectively.

Then there are two open neighborhoods $A_{0}$ and $A_{1}$ of $S$ in $E$ such that there exists an isomorphism $\left(\mathrm{BFV}^{A_{0}}(E),[\cdot, \cdot]_{\mathrm{BFV}}^{0, A_{0}}\right) \stackrel{\cong}{\longrightarrow}\left(\mathrm{BFV}^{A_{1}}(E),[\cdot, \cdot]_{\mathrm{BFV}}^{1, A_{1}}\right)$ of graded Poisson algebras.

Proof. We use the fact that any two embeddings of $E$ as a tubular neighborhood are homotopic up to inner automorphisms of $E$; that is, given two embeddings $\psi$ and $\varphi$ of $E$ into $M$ as a tubular neighborhood, one can find

- a vector bundle isomorphism $g$ of $E$ and

- a smooth map $F: E \times I \rightarrow M$

such that

- $\left.F\right|_{E \times\{0\}}=\psi$ and $\left.F\right|_{E \times\{1\}}=\varphi \circ g$,

- $\psi_{s}:=\left.F\right|_{E \times\{s\}}: E \rightarrow M$ is an embedding for all $s \in I$, and

- $\left.\psi_{s}\right|_{S}=\operatorname{id}_{S}$ for all $s \in I$.

The construction of $F$ can be found in [Hirsch 1994], for instance.

Since vector-bundle automorphisms of $E$ yield isomorphic BVF complexes by Lemma 4.1, we can assume without loss of generality that the two embeddings $\psi:=\psi_{0}$ and $\varphi=: \psi_{1}$ are homotopic (that is, $g=\mathrm{id}$ ).

Denote the images of the $\psi_{s}$ by $V_{s}$. Since $\psi_{s}$ is an embedding of a manifold of the same dimension as $M$, the image $V_{s}$ is an open subset of $M$. Moreover, $S \subset V_{s}$ holds for arbitrary $s \in I$; that is, $V_{s}$ is an open neighborhood of $S$ in $M$. Because $F$ is continuous, one can find an open neighborhood $V$ of $S$ in $M$ that is contained in $\bigcap_{s \in I} V_{s}$.

We define $\hat{F}: E \times I \rightarrow M \times I$ by $(e, t) \mapsto(F(e, t), t)$ and check that $\hat{F}$ is an embedding and hence that its image is a submanifold $W$ of $M \times I$ and $\hat{F}$ is a diffeomorphism between $E \times I$ and $W$. We then consider the restriction of $\hat{F}^{-1}: W \stackrel{\cong}{\rightrightarrows} E \times I$ to $V \times I$, which we denote by $G$. If one restricts $G$ to slices of the form $V \times\{s\}$, one obtains $\left.\psi_{s}^{-1}\right|_{V}$. The images of $\left.\psi_{s}^{-1}\right|_{V}$ are denoted by $W_{s}$. By the continuity of $G$, there is an open neighborhood $W$ of $S$ in $E$ that is contained in $\bigcap_{s \in I} W_{s}$.

We define the one-parameter family

$$
\varphi_{s}: W_{0} \stackrel{\left.\psi_{0}\right|_{W_{0}}}{\longrightarrow} V \stackrel{\left(\left.\psi_{s}\right|_{V}\right)^{-1}}{\longrightarrow} W_{s}
$$


of local diffeomorphisms of $E$. Moreover, $E$ inherits a one-parameter family of Poisson bivector fields defined by $\Pi_{s}:=\left(\left.\psi_{s}\right|_{V_{s}} ^{-1}\right)_{*}\left(\left.\Pi\right|_{V_{s}}\right)$. The restriction $\left.\Pi_{s}\right|_{W_{s}}$ is equal to $\left(\left.\psi_{s}\right|_{V} ^{-1}\right)_{*}\left(\left.\Pi\right|_{V}\right)$. Consequently

$$
\left.\Pi_{s}\right|_{W_{s}}=\left(\varphi_{s}\right)_{*}\left(\left.\Pi_{0}\right|_{W_{0}}\right) \quad \text { for all } s \in I .
$$

Differentiating $\varphi_{s}$ yields a smooth one-parameter family of local vector fields $\left(Y_{s}\right)_{s \in I}$ on $E$. By equation (1), the smooth one-parameter family $\left.\Pi_{t}\right|_{W}-\left.Y_{t}\right|_{W} d t$ is an MC element of $\left(\mathscr{V}(W)[1] \otimes \Omega(I), d_{D R},[\cdot, \cdot]_{\mathrm{SN}}\right)$.

The $L_{\infty}$ quasi-isomorphism

$$
\mathscr{L}_{\nabla}:\left(\mathscr{V}(E)[1],[\cdot, \cdot]_{\mathrm{SN}}\right) \rightarrow\left(\mathscr{V}\left(\mathscr{E}^{*}[1] \oplus \mathscr{E}[-1]\right)[1],[G, \cdot]_{\mathrm{SN}},[\cdot, \cdot]_{\mathrm{SN}}\right)
$$

from Proposition 2.3 restricts to an $L_{\infty}$ quasi-isomorphism

$$
\left.\mathscr{L}_{\nabla}\right|_{W}:\left(\mathscr{V}(W)[1],[\cdot, \cdot]_{\mathrm{SN}}\right) \rightarrow\left(\mathscr{V}\left(\left.\left(\mathscr{E}^{*}[1] \oplus \mathscr{E}[-1]\right)\right|_{W}\right)[1],[G, \cdot]_{\mathrm{SN}},[\cdot, \cdot]_{\mathrm{SN}}\right) .
$$

Hence we obtain an $L_{\infty}$ quasi-isomorphism

$\left.\mathscr{L}_{\nabla}\right|_{W} \otimes \mathrm{id}:\left(\mathscr{V}(W)[1] \otimes \Omega(I), d_{D R},[\cdot, \cdot]_{\mathrm{SN}}\right)$

$$
\rightarrow\left(\mathscr{V}\left(\left.\mathscr{E}^{*}[1] \oplus \mathscr{E}[-1]\right|_{W}\right)[1] \otimes \Omega(I), d_{D R}+[G, \cdot]_{\mathrm{SN}},[\cdot, \cdot]_{\mathrm{SN}}\right) .
$$

Applying $\left.\mathscr{L}_{\nabla}\right|_{W} \otimes$ id to the MC element $\left.\Pi_{t}\right|_{W}-\left.Y_{t}\right|_{W} d t$ and adding $G$ yields an MC element $\hat{\Pi}_{t}-\hat{Y}_{t} d t$ of $\left(\mathscr{V}\left(\left.\mathscr{E}^{*}[1] \oplus \mathscr{E}[-1]\right|_{W}\right)[1] \otimes \Omega(I), d_{D R},[\cdot, \cdot]_{\mathrm{SN}}\right)$.

It is straightforward to check that $\hat{\Pi}_{s}$ is the restriction of $\mathscr{L}_{\nabla}\left(\sum_{k \geq 1} 1 /(k !) \Pi_{s}^{\otimes k}\right)$ to $W$ and that $\hat{Y}_{S}$ is the sum of the horizontal lift $\iota_{\nabla}\left(Y_{S}\right)$ of $Y_{S}$ (with respect to $\nabla$ ) restricted to $W$ plus a part in $\mathscr{V}^{(1,1)}\left(\mathscr{E}^{*}[1] \oplus \mathscr{E}[-1]\right.$ ) (which acts as a nilpotent derivation).

Using parallel transport with respect to $\nabla$, we can integrate $\left(\iota_{\nabla}\left(Y_{t}\right)\right)_{t \in I}$ to a one-parameter family of vector-bundle automorphisms

$$
\hat{\varphi}_{s}:\left.\left.\mathscr{E}^{*}[1] \oplus \mathscr{E}[-1]\right|_{W_{0}} \rightarrow \mathscr{E}^{*}[1] \oplus \mathscr{E}[-1]\right|_{W_{s}}
$$

covering $\varphi_{s}: W_{0} \rightarrow W_{s}$ for arbitrary $s \in I$. In a way similar to the construction of $V$ and $W$, one finds an open neighborhood $A_{0}$ of $S$ in $W$ such that

$$
\left.\varphi_{t}\right|_{A_{0}}: A_{0} \stackrel{\cong}{\longrightarrow} A_{t}
$$

with $\bigcup_{s \in I} A_{s} \subset W$. Therefore the restriction of $\hat{\varphi}_{s}$ to $\left.\mathscr{E}^{*}[1] \oplus \mathscr{E}[-1]\right|_{A_{0}}$ has image $\left.\mathscr{E}^{*}[1] \oplus \mathscr{E}[-1]\right|_{A_{s}}$, which is a submanifold of $\left.\mathscr{E}^{*}[1] \oplus \mathscr{E}[-1]\right|_{W}$ for arbitrary $s \in I$.

Hence the one-parameter family

$$
\left(\left.\iota_{\nabla}\left(Y_{t}\right)\right|_{\left.(\mathscr{E} *[1] \oplus \mathscr{E}[-1])\right|_{A_{t}}}\right)_{t \in I}
$$

of local vector fields can be uniquely integrated to a one-parameter family of local diffeomorphisms $\left(\hat{\varphi}_{t}\right)_{t \in I}$, and therefore the one-parameter family of local vector 
fields $\left(\left.\hat{Y}_{t}\right|_{A_{t}}\right)_{t \in I}$ can be uniquely integrated to a one-parameter family of local diffeomorphisms, which we denote by

$$
\varphi_{s}:\left.\left.\left(\mathscr{E}^{*}[1] \oplus \mathscr{\mathscr { E }}[-1]\right)\right|_{A_{0}} \rightarrow\left(\mathscr{E}^{*}[1] \oplus \mathscr{E}[-1]\right)\right|_{A_{s}} \quad \text { for } s \in I .
$$

Applying Lemma 2.1 shows that $\left.\hat{\Pi}_{s}\right|_{A_{s}}=\left(\varphi_{s}\right)_{*}\left(\left.\hat{\Pi}_{0}\right|_{A_{0}}\right)$ holds for all $s \in I$. Hence

$$
\left(\varphi_{1}\right)_{*}: \mathscr{C}^{\infty}\left(\left.\mathscr{E}^{*}[1] \oplus \mathscr{E}[-1]\right|_{A_{0}}\right) \rightarrow \mathscr{C}^{\infty}\left(\left.\mathscr{E}^{*}[1] \oplus \mathscr{E}[-1]\right|_{A_{1}}\right)
$$

is an isomorphism of Poisson algebras.

Theorem 4.4. Let $S$ be a coisotropic submanifold of a smooth, finite-dimensional Poisson manifold $(M, \Pi)$. Suppose

$$
\left(\mathrm{BFV}(E), D_{\mathrm{BFV}}^{0},[\cdot, \cdot]_{\mathrm{BFV}}^{0}\right) \text { and }\left(\mathrm{BFV}(E), D_{\mathrm{BFV}}^{1},[\cdot, \cdot]_{\mathrm{BFV}}^{1}\right)
$$

are two BFV complexes constructed with the help of two arbitrary embeddings of $E$ into $M$, two arbitrary connections on $E \rightarrow S$, and two arbitrary BFV charges.

Then there are two open neighborhoods $B_{0}$ and $B_{1}$ of $S$ in $E$ such that there exists an isomorphism

$$
\left(\mathrm{BFV}^{B_{0}}(E), D_{\mathrm{BFV}}^{0, B_{0}},[\cdot, \cdot]_{\mathrm{BFV}}^{0, B_{0}}\right) \stackrel{\cong}{\longrightarrow}\left(\mathrm{BFV}^{B_{1}}(E), D_{\mathrm{BFV}}^{1, B_{1}},[\cdot, \cdot]_{\mathrm{BFV}}^{1, B_{1}}\right) .
$$

of differential graded Poisson algebras

Proof. By Theorem 3.4, we can assume without loss of generality that the two chosen connections coincide. It suffices to prove that there is an isomorphism of graded Poisson algebras from some restriction of $\left.\operatorname{BFV}(E),[\cdot, \cdot]_{\mathrm{BFV}}^{0}\right)$ to some restriction of $\left(\mathrm{BFV}(E),[\cdot, \cdot]_{\mathrm{BFV}}^{0}\right)$ that maps a restricted $\mathrm{BFV}$ charge to another restricted BFV charge. This is a consequence of the fact that Theorem 3.4 holds also in the restricted setting as long as the open neighborhood $U$ of $S$ in $E$ that we restrict to is contractible to $S$ along the fibers of $E$.

By Lemma 4.1, we may assume without loss of generality that the two embeddings we consider are homotopic. Hence there is a smooth one-parameter family of isomorphisms of graded Poisson algebras

$$
\left(\varphi_{s}\right)_{*}:\left(\mathrm{BFV}^{A_{0}}(E),[\cdot, \cdot]_{\mathrm{BFV}}^{0, A_{0}}\right) \rightarrow\left(\mathrm{BFV}^{A_{s}}(E),[\cdot, \cdot]_{\mathrm{BFV}}^{s, A_{s}}\right),
$$

which we constructed in the proof of Proposition 4.3. The smoothness of this family and the fact that the zero section $S$ is fixed under $\left(\varphi_{s}\right)_{s \in I}$ imply there is a open neighborhood $A$ of $S$ in $E$ such that $A \subset \bigcap_{s \in I} A_{s}$.

Fix a restricted BFV charge $\Omega$ of $\left(\operatorname{BFV}^{A_{0}}(E),[\cdot, \cdot]_{\mathrm{BFV}}^{0, A_{0}}\right)$. The restriction of

$$
\left(\Omega(t):=\left(\varphi_{t}\right)_{*}(\Omega)\right)_{t \in I}
$$

to $A$ yields a smooth one-parameter family of sections of $\left.\bigwedge^{\mathscr{E}} \otimes \bigwedge^{\mathscr{E}}{ }^{*}\right|_{A}$. Although $\left[\left.\Omega(s)\right|_{A},\left.\Omega(s)\right|_{A}\right]_{\mathrm{BFV}}^{s, A}=0$ for all $s \in I$, the restriction $\left.\Omega(s)\right|_{A}$ is in general not a 
BFV charge since its component in $\Gamma\left(\left.\mathscr{E}\right|_{W}\right)$ is $\Omega_{0}(s):=\left(\varphi_{s}\right)_{*}\left(\Omega_{0}\right)$, which does not need to be equal to $\Omega_{0}$ as required; see Definition 4.2. In particular, $\Omega(1)$ might not be a restricted $\mathrm{BFV}$ charge of $\left.\operatorname{BFV}(E),[\cdot, \cdot]_{\mathrm{BFV}}^{1}\right)$. However, in the remainder of the proof, we will show that $\Omega(1)$ can be gauged to a BFV charge.

We recall some of the ingredients of the proof of Proposition 2.5. First observe that $\delta:=\left[\Omega_{0}, \cdot\right]_{G}$ is a differential. Here we denote by $\Omega_{0}$ the tautological section of $\mathscr{E} \rightarrow E$, by $G$ the Poisson bivector field associated to the fiber pairing between $\mathscr{E}$ and $\mathscr{E}^{*}$, and by $[\cdot, \cdot]_{G}$ the graded Poisson bracket on $\operatorname{BFV}(E)$ corresponding to $G$. Second, it is possible to construct a homotopy $h$ for $\delta$, that is, a degree -1 map satisfying

$$
\delta \circ h+h \circ \delta=\mathrm{id}-i \circ \mathrm{pr},
$$

where $i$ is an embedding of the cohomology of $\delta$ into $\operatorname{BFV}(E)$, and pr is a projection from $\mathrm{BFV}(E)$ onto cohomology. We remark that $h$ does not restrict to arbitrary open neighborhoods of $S$ in $E$. However, one can check that it does restrict to open neighborhoods that can be contracted to $S$ along the fibers of $E$. Without loss of generality, we can assume that $A$ has this property.

We are interested in the smooth one-parameter family

$$
h\left(\Omega_{0}(s)\right) \in \Gamma\left(\left.\mathscr{E} \otimes \mathscr{E}^{*}\right|_{A}\right) \cong \Gamma\left(\operatorname{End}\left(\left.\mathscr{E}\right|_{A}\right)\right) \quad \text { for } s \in I .
$$

Since $\Omega_{0}$ intersects the zero section of $\mathscr{E} \rightarrow E$ transversally at $S$, so does $\Omega_{0}(s)$ for arbitrary $s \in I$. This implies first that the evaluation of $\Omega_{0}(s)$ at $S$ is zero, and second that $\left.h\left(\Omega_{0}(s)\right)\right|_{S} \in \Gamma\left(\left.\mathscr{E} \otimes \mathscr{E}^{*}\right|_{S}\right)$ is fiberwise invertible; that is, it is an element of $\Gamma\left(G L\left(\left.\mathscr{E}\right|_{S}\right)\right)$.

For any $s \in I$, we have $\delta\left(\Omega_{0}(s)\right)=\left[\Omega_{0}, \Omega_{0}(s)\right]_{G}=0$ since both $\Omega_{0}$ and $\Omega_{0}(s)$ are sections of $\left.\mathscr{E}\right|_{A}$ and $G$ is the Poisson bivector given by contraction between $\mathscr{E}$ and $\mathscr{E}^{*}$. Moreover, $(i \circ \mathrm{pr})\left(\Omega_{0}(s)\right)=0$ since the projection pr involves evaluation of the section at $S$, where $\Omega_{0}(s)$ vanishes. Consequently, equation (2) reduces to $\delta\left(h\left(\Omega_{0}(s)\right)\right)=\Omega_{0}(s)$ for all $s \in I$. However, this means that if we interpret $h\left(\Omega_{0}(s)\right)$ as a fiberwise endomorphism of $\left.\mathscr{E}\right|_{A}$, the image of $\Omega_{0}$ under $-h\left(\Omega_{0}(s)\right)$ is $\Omega_{0}(s)$.

We define $M_{s}:=-h\left(\Omega_{0}(s)\right)$. As already observed, $\left(M_{t}\right)_{t \in I}$ is a smooth oneparameter family of sections of $\operatorname{End}\left(\left.\mathscr{E}\right|_{A}\right)$, and the restriction to $S$ is a smooth one-parameter family of GL( $(\mathscr{E} \mid S)$. By smoothness of the one-parameter family, it is possible to find an open neighborhood $B$ of $S$ in $E$ such that the restriction of $\left(M_{t}\right)_{t \in I}$ to $B$ is always fiberwise invertible. Since $M_{0}=\left.\mathrm{id}\right|_{A}$, we know that $\left(\left.M_{t}\right|_{B}\right)_{t \in I}$ is a smooth one-parameter family of sections in $\mathrm{GL}_{+}\left(\left.\mathscr{E}\right|_{B}\right)$, that is, fiberwise invertible automorphisms of $\left.E\right|_{B}$ with positive determinant. In particular $M_{1} \in \Gamma\left(\mathrm{GL}_{+}\left(\left.\mathscr{E}\right|_{B}\right)\right)$. 
Consider the smooth one-parameter family $\left(m_{t}\right)_{t \in I}$ of sections of $\operatorname{End}\left(\left.\mathscr{E}\right|_{B}\right)$ given by

$$
m_{t}:=-M_{t}^{-1} \circ\left(\frac{d}{d t} M_{t}\right) .
$$

It integrates to a smooth one-parameter family of sections of $\mathrm{GL}_{+}\left(\left.\mathscr{E}\right|_{B}\right)$ that coincides with $\left(M_{t}\right)_{t \in[0,1]}$. The adjoint action of $m_{t}$ on $\left(\mathrm{BFV}^{B}(E),[\cdot, \cdot]_{\mathrm{BFV}}^{1, B}\right)$ can be integrated to an automorphism of $\left(\operatorname{BFV}^{B}(E),[\cdot, \cdot]_{\mathrm{BFV}}^{1, B}\right)$, and this automorphism maps the restriction of $\Omega_{0}(1)$ to $B$ to the restriction of $\Omega_{0}$ to $B$. Hence $\exp (m) \circ\left(\varphi_{1}\right)_{*}$ maps the restricted BFV charge $\Omega$ to another restricted BFV charge of $\left(\mathrm{BFV}^{B}(E),[\cdot, \cdot]_{\mathrm{BFV}}^{1, B}\right)$.

Definition 4.5. Let $\left(\mathrm{BFV}(E), D_{\mathrm{BFV}},[\cdot, \cdot]_{\mathrm{BFV}}\right)$ be a BFV complex associated to a coisotropic submanifold $S$ of a smooth Poisson manifold $(M, \Pi)$. We define a differential graded Poisson algebra $\left(\operatorname{BFV}^{\mathfrak{g}}(E), D_{\mathrm{BFV}}^{\mathfrak{g}},[\cdot, \cdot]_{\mathrm{BFV}}^{\mathfrak{g}}\right)$ as follows:

(a) $\operatorname{BFV}^{\mathfrak{g}}(E)$ is the algebra of equivalence classes of elements of $\operatorname{BFV}(E)$ under the equivalence relation $f \sim g$ if and only if there is a open neighborhood $U$ of $S$ in $E$ such that $\left.f\right|_{U}=\left.g\right|_{U}$;

(b) $D_{\mathrm{BFV}}^{\mathfrak{g}}([\cdot]):=\left[D_{\mathrm{BFV}}(\cdot)\right]$, where $[\cdot]$ denotes the class of $\cdot$ under $\sim$;

(c) $[[\cdot],[\cdot]]_{\mathrm{BFV}}^{\mathfrak{g}}:=\left[[\cdot, \cdot]_{\mathrm{BFV}}\right]$.

Given a differential graded Poisson algebra with unit $(A, \wedge, d,[\cdot, \cdot])$, we define the corresponding abstract differential graded Poisson algebra $[(A, \wedge, d,[\cdot, \cdot])]$ with unit to be the isomorphism class of $(A, \wedge, d,[\cdot, \cdot])$ in the category of differential graded Poisson algebras with unit. In particular, $[(A, \wedge, d,[\cdot, \cdot])]$ is an object in the category of differential graded Poisson algebras with unit, up to isomorphisms.

Theorem 4.4 has an immediate corollary:

Corollary 4.6. Consider a coisotropic submanifold $S$ of a smooth, finite-dimensional Poisson manifold $(M, \Pi)$, and let $\left(\mathrm{BFV}(E), D_{\mathrm{BFV}},[\cdot, \cdot]_{\mathrm{BFV}}\right)$ be a BFV complex associated to $S$ inside $(M, \Pi)$.

The abstract differential graded Poisson algebra

$$
\left[\left(\operatorname{BFV}^{\mathfrak{g}}(E), D_{\mathrm{BFV}}^{\mathfrak{g}},[\cdot, \cdot]_{\mathrm{BFV}}^{\mathfrak{g}}\right)\right]
$$

is independent of the specific choice of a BFV complex and hence is an invariant of $S$ as a coisotropic submanifold of $(M, \Pi)$.

\section{Acknowledgments}

I thank Alberto Cattaneo for remarks on a draft of this work. Moreover I thank the referee for helpful comments. 


\section{References}

[Batalin and Fradkin 1983] I. A. Batalin and E. S. Fradkin, "A generalized canonical formalism and quantization of reducible gauge theories", Phys. Lett. B 122:2 (1983), 157-164. MR 85b:81080 Zbl 0967.81508

[Batalin and Vilkovisky 1977] I. A. Batalin and G. S. Vilkovisky, "Relativistic S-matrix of dynamical systems with bosons and fermion constraints", Phys. Lett. 69B (1977), 309-312.

[Bordemann 2000] M. Bordemann, "The deformation quantization of certain super-Poisson brackets and BRST cohomology", preprint, 2000. arXiv math.QA/0003218

[Costello 2007] K. J. Costello, "Renormalisation and the Batalin-Vilkovisky formalism", preprint, 2007. arXiv 0706.1533

[Gugenheim and Lambe 1989] V. K. A. M. Gugenheim and L. A. Lambe, "Perturbation theory in differential homological algebra, I", Illinois J. Math. 33:4 (1989), 566-582. MR 91e:55023 Zbl 0661.55018

[Herbig 2006] H.-C. Herbig, Variations on homological reduction, Ph.D. thesis, Goethe Universität Frankfurt am Main, 2006. Zbl 1196.81149 arXiv 0708.3598

[Hirsch 1994] M. W. Hirsch, Differential topology, Graduate Texts in Mathematics 33, Springer, New York, 1994. MR 96c:57001 Zbl 0356.57001

[Markl et al. 2002] M. Markl, S. Shnider, and J. Stasheff, Operads in algebra, topology and physics, Mathematical Surveys and Monographs 96, American Mathematical Society, Providence, RI, 2002. MR 2003f:18011 Zbl 1017.18001

[Schätz 2009a] F. Schätz, "BFV-complex and higher homotopy structures", Comm. Math. Phys. 286:2 (2009), 399-443. MR 2010h:53127

[Schätz 2009b] F. Schätz, "Moduli of coisotropic sections and the BFV-complex", preprint, 2009. arXiv 0903.4074

[Stasheff 1997] J. Stasheff, "Homological reduction of constrained Poisson algebras", J. Differential Geom. 45:1 (1997), 221-240. MR 98i:17026 Zbl 0874.58020

[Weinstein 1988] A. Weinstein, "Coisotropic calculus and Poisson groupoids", J. Math. Soc. Japan 40:4 (1988), 705-727. MR 90b:58091 Zbl 0642.58025

Received July 14, 2009. Revised September 12, 2010.

\section{FLORIAN SCHÄTZ}

Center for Mathematical Analysis, Geometry and Dynamical Systems

DePartamento de Matematica

AV. ROVISCO PAIS

1049-001 LISBON

PORTUGAL

florian.schaetz@gmail.com 


\title{
PACIFIC JOURNAL OF MATHEMATICS
}

\author{
http://www.pjmath.org \\ Founded in 1951 by \\ E. F. Beckenbach (1906-1982) and F. Wolf (1904-1989)
}

\section{EDITORS}

V. S. Varadarajan (Managing Editor)

Department of Mathematics

University of California

Los Angeles, CA 90095-1555

pacific@math.ucla.edu

Vyjayanthi Chari

Department of Mathematics

University of California

Riverside, CA 92521-0135

chari@math.ucr.edu

Robert Finn

Department of Mathematics Stanford University

Stanford, CA 94305-2125

finn@math.stanford.edu

Kefeng Liu

Department of Mathematics

University of California

Los Angeles, CA 90095-1555

liu@math.ucla.edu
Darren Long

Department of Mathematics

University of California

Santa Barbara, CA 93106-3080

long@math.ucsb.edu

Jiang-Hua Lu

Department of Mathematics

The University of Hong Kong

Pokfulam Rd., Hong Kong jhlu@maths.hku.hk

Alexander Merkurjev

Department of Mathematics

University of California

Los Angeles, CA 90095-1555

merkurev@math.ucla.edu
Sorin Popa

Department of Mathematics University of California

Los Angeles, CA 90095-1555 popa@math.ucla.edu

Jie Qing

Department of Mathematics

University of California

Santa Cruz, CA 95064

qing@cats.ucsc.edu

Jonathan Rogawski

Department of Mathematics

University of California

Los Angeles, CA 90095-1555

jonr@math.ucla.edu

\section{PRODUCTION}

pacific@math.berkeley.edu

\begin{abstract}
Silvio Levy, Scientific Editor Matthew Cargo, Senior Production Editor
\end{abstract}
ACADEMIA SINICA, TAIPEI

CALIFORNIA INST. OF TECHNOLOGY

INST. DE MATEMÁTICA PURA E APLICADA

KEIO UNIVERSITY

MATH. SCIENCES RESEARCH INSTITUTE

NEW MEXICO STATE UNIV.

OREGON STATE UNIV.

\section{SUPPORTING INSTITUTIONS}

STANFORD UNIVERSITY
UNIV. OF BRITISH COLUMBIA
UNIV. OF CALIFORNIA, BERKELEY
UNIV. OF CALIFORNIA, DAVIS
UNIV. OF CALIFORNIA, LOS ANGELES
UNIV. OF CALIFORNIA, RIVERSIDE
UNIV. OF CALIFORNIA, SAN DIEGO
UNIV. OF CALIF., SANTA BARBARA

UNIV. OF CALIF., SANTA CRUZ

UNIV. OF MONTANA

UNIV. OF OREGON

UNIV. OF SOUTHERN CALIFORNIA

UNIV. OF UTAH

UNIV. OF WASHINGTON

WASHINGTON STATE UNIVERSITY

These supporting institutions contribute to the cost of publication of this Journal, but they are not owners or publishers and have no responsibility for its contents or policies.

See inside back cover or www.pjmath.org for submission instructions.

The subscription price for 2010 is US \$420/year for the electronic version, and \$485/year for print and electronic.

Subscriptions, requests for back issues from the last three years and changes of subscribers address should be sent to Pacific Journal of Mathematics, P.O. Box 4163, Berkeley, CA 94704-0163, U.S.A. Prior back issues are obtainable from Periodicals Service Company, 11 Main Street, Germantown, NY 12526-5635. The Pacific Journal of Mathematics is indexed by Mathematical Reviews, Zentralblatt MATH, PASCAL CNRS Index, Referativnyi Zhurnal, Current Mathematical Publications and the Science Citation Index.

The Pacific Journal of Mathematics (ISSN 0030-8730) at the University of California, c/o Department of Mathematics, 969 Evans Hall, Berkeley, CA 94720-3840, is published monthly except July and August. Periodical rate postage paid at Berkeley, CA 94704, and additional mailing offices. POSTMASTER: send address changes to Pacific Journal of Mathematics, P.O. Box 4163, Berkeley, CA 94704-0163.

PJM peer review and production are managed by EditFLOW ${ }^{\mathrm{TM}}$ from Mathematical Sciences Publishers.

PUBLISHED BY PACIFIC JOURNAL OF MATHEMATICS

at the University of California, Berkeley 94720-3840

A NON-PROFIT CORPORATION

Typeset in LATEX

Copyright $(\mathrm{C} 2010$ by Pacific Journal of Mathematics 


\section{PACIFIC JOURNAL OF MATHEMATICS}

Volume $248 \quad$ No. $2 \quad$ December 2010

Topological description of Riemannian foliations with dense leaves

Jesús A. ÁlVAREZ LóPEZ and Alberto CANDEL

The nonexistence of quasi-Einstein metrics

JEFFREY S. CASE

Twisted symmetric group actions

AKINARI HoSHI and MING-CHANG KANG

Optimal transportation and monotonic quantities on evolving manifolds

305

HONG HUANG

Hopf structures on the Hopf quiver $Q(\langle g\rangle, g)$

HuA-Lin HuANG, YU Ye and QING ZHaO

Minimal surfaces in $S^{3}$ foliated by circles

Nikolai KuteV and VelichKa Milousheva

Prealternative algebras and prealternative bialgebras

XIANG Ni and CHENGMING BAI

Some remarks about closed convex curves

Ke OU and Shengliang PAN

Orbit correspondences for real reductive dual pairs

SHU-YEN PAN

Graphs of bounded degree and the $p$-harmonic boundary

Michael J. Puls

Invariance of the BFV complex

FLORIAN SCHÄTZ

Some elliptic PDEs on Riemannian manifolds with boundary

YANNICK SIRE and ENRICO VALDINOCI

Representations of Lie superalgebras in prime characteristic, III 